\title{
Investigating the spectral characteristics of backscattering from heterogeneous spherical nuclei using broadband finite-difference time-domain simulations
}

\author{
Guo-Shan Chao \\ National Taiwan University \\ Graduate Institute of Biomedical Electronics and \\ Bioinformatics \\ No. 1, Section 4 Roosevelt Road \\ Taipei, 10617 Taiwan
}

\author{
Kung-Bin Sung \\ National Taiwan University \\ Graduate Institute of Biomedical Electronics and \\ Bioinformatics \\ and \\ Department of Electrical Engineering \\ No. 1, Section 4 Roosevelt Road \\ Taipei, 10617 Taiwan
}

\begin{abstract}
Reflectance spectra measured from epithelial tissue have been used to extract size distribution and refractive index of cell nuclei for noninvasive detection of precancerous changes. Despite many in vitro and in vivo experimental results, the underlying mechanism of sizing nuclei based on modeling nuclei as homogeneous spheres and fitting the measured data with Mie theory has not been fully explored. We describe the implementation of a three-dimensional finitedifference time-domain (FDTD) simulation tool using a Gaussian pulse as the light source to investigate the wavelength-dependent characteristics of backscattered light from a nuclear model consisting of a nucleolus and clumps of chromatin embedded in homogeneous nucleoplasm. The results show that small-sized heterogeneities within the nuclei generate about five times higher backscattering than homogeneous spheres. More interestingly, backscattering spectra from heterogeneous spherical nuclei show periodic oscillations similar to those from homogeneous spheres, leading to high accuracy of estimating the nuclear diameter by comparison with Mie theory. In addition to the application in light scattering spectroscopy, the reported FDTD method could be adapted to study the relations between measured spectral data and nuclear structures in other optical imaging and spectroscopic techniques for in vivo diagnosis. () 2010 Society of PhotoOptical Instrumentation Engineers. [DOI: 10.1117/1.3324838]
\end{abstract}

Keywords: light scattering spectroscopy; finite-difference time-domain; Mie theory; nucleus; dysplasia.

Paper 09247R received Jun. 12, 2009; revised manuscript received Nov. 9, 2009; accepted for publication Jan. 6, 2010; published online Feb. 24, 2010.

\section{Introduction}

Elastic light scattering can be used to extract size and refractive index distributions of scattering structures in biological cells and tissue. ${ }^{1-7}$ While both angular and wavelengthdependent characteristics of scattered light can be used to estimate the size of cell nuclei, the wavelength dependence of backscattering is easier to measure from cells/tissue in vivo and more suitable for noninvasive detection of early changes associated with cancer. ${ }^{3-6}$ Size estimation is typically achieved by fitting the experimental data with analytical solutions provided by Mie theory in which light scattering objects are treated as homogeneous spheres. ${ }^{1-7}$ However, light scattering characteristics of nuclei are greatly influenced by nuclear morphology and heterogeneities within the nuclei, and may deviate appreciably from predictions based on Mie theory. ${ }^{8}$ Experimental measurements of the angular dependence of polarized scattering from whole cells and isolated

Address all correspondence to: Kung-Bin Sung, National Taiwan University, Department of Electrical Engineering, No. 1, Section 4 Roosevelt Road, Taipei, 10617 Taiwan. Tel: 886-2-33669675; Fax: 886-2-33669630; E-mail: kbsung@cc.ee.ntu.edu.tw nuclei differed greatly from values calculated with a model assuming homogeneous scatterers. ${ }^{2}$ Despite inaccurate modeling of nuclei as homogeneous spheres, results of using Miebased models to size nuclei from backscattering spectra were confirmed in vitro with light microscopy measurements. ${ }^{4,6,9}$

One powerful tool to address this limitation is the finitedifference time-domain (FDTD) method, which provides rigorous solutions to Maxwell's equations numerically. The FDTD method allows arbitrary geometry and refractive index distribution of scatterers to be simulated in the light scattering problem, and has been used to investigate angular scattering characteristics of biological cells. ${ }^{8,10-14}$ The spectral features of backscattering from cells, despite being more accessible in vivo than the angular dependence, have not been investigated systematically using the FDTD method. ${ }^{15}$ There exist related studies focused on exploring the wavelength dependence of the total scattering cross sections of inhomogeneous spheres ${ }^{16}$ and irregularly shaped homogeneous particles ${ }^{17}$ using both equiphase-sphere approximation and broadband FDTD simulation. A thorough review can be found in Ref. 18

$1083-3668 / 2010 / 15(1) / 015007 / 6 / \$ 25.00$ ○ 2010 SPIE 
We present the results of investigating the influences of heterogeneous refractive index distribution of nuclei on the spectral characteristics of backscattered light using the broadband FDTD method. In particular, we aim to examine the validity of the widely used method for estimating the size of nuclei based on Mie theory. Our long term goals are to increase the understanding of the relationships between light scattering and cellular structures, and use the knowledge to facilitate the development of noninvasive or minimally invasive diagnostic tools for early detection of cancer. As an initial effort, we focus on cells with low mitochondrial contents such as cervical epithelial cells. ${ }^{8,12}$ In vivo reflectance confocal imaging of cervical epithelium confirmed that the cell nucleus is a major source of contrast, indicating that the nucleus has a larger cross section for backscattering than other organelles and cytoplasmic membranes. ${ }^{19}$

\section{Methods}

The FDTD method, proposed by Yee in $1966,{ }^{20}$ provides numerical solutions of Maxwell's equations through discretization of the electric and magnetic fields in both time and spatial domains. To investigate the spectral characteristics of the backscattered light efficiently, we adopted the method proposed by Drezek, Dunn, and Richards-Kortum that used a Gaussian pulse as a broadband light source and generated angular scattering patterns at multiple wavelengths with one FDTD run. ${ }^{15}$ To enhance the dynamic range, we modulated the Gaussian pulse with a sinusoidal wave to shift the central wavelength of the light source to $1200 \mathrm{~nm}$, the shortest wavelength simulated. Spectral data were obtained in wavelengths ranging from 1200 to $1400 \mathrm{~nm}$ with a 10-nm increment, considering future development of optical systems for in vivo applications. We used a grid size of $44.118 \mathrm{~nm}$, corresponding to $1 / 20$ of the shortest wavelength in the medium, as a result of trade-off between accuracy and computational time. A perfectly matched layer (PML) absorbing boundary condition was implemented for suppressing artificial reflection. ${ }^{21}$ The intensity of scattered light was integrated over the whole $2 \pi$ azimuthal angles and the scattering angles between 164 and $180 \mathrm{deg}$, assuming plane wave illumination and a waterimmersion objective lens with a numerical aperture (NA) of 0.4 for collecting the backscattered light. Our 3-D FDTD code was implemented in $\mathrm{C} / \mathrm{C}++$ on a personal computer with an Intel $3.16 \mathrm{GHz}$ Core $^{\mathrm{TM}} 2$ Duo processor and $8 \mathrm{~GB}$ of memory.

Cell nuclei were modeled as 5- $\mu \mathrm{m}$ spheres consisting of a spherical nucleolus and clumps of chromatin randomly distributed in a homogeneous medium that represents the cytoplasm. The diameter of the nucleolus was set to be either 1.25 or $1.67 \mu \mathrm{m}$. The size of each chromatin clump was assigned randomly in the range of 0.033 to $1 \mu \mathrm{m}$. A higher density of chromatin condensation was distributed at the periphery of the nucleus according to scanning electron microscopic images of mammalian cells. ${ }^{22}$ We note that the structure of refractive index fluctuation in biological tissue and cells has been suggested to exhibit self-affine or fractal features, ${ }^{7,23-27}$ and this information was not incorporated into our model (see the discussion in Sec. 4 for details).

The refractive index of the cytoplasm was set at 1.36 according to reported measurements, ${ }^{6,8,28}$ and the nucleoplasm
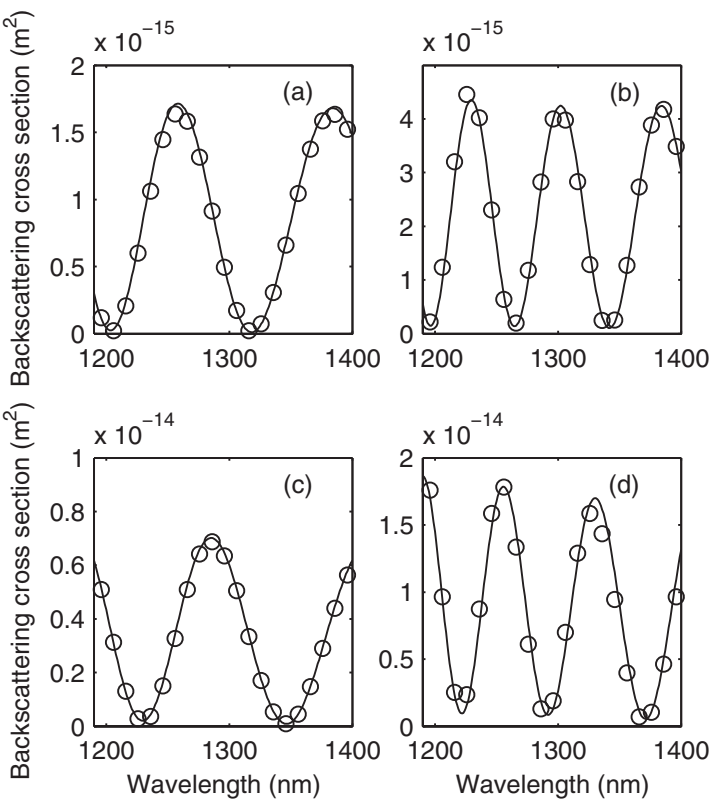

Fig. 1 Backscattering spectra of homogeneous spheres by FDTD (circles) and Mie theory (lines). The spheres have refractive indices of (a) and (b) 1.39 and (c) and (d) 1.42, and diameters of (a) and (c) $5 \mu \mathrm{m}$ and (b) and (d) $8 \mu \mathrm{m}$.

was assumed to have the same refractive index as the cytoplasm. ${ }^{29}$ The average refractive index of the nucleolus and chromatin condensation for normal cells was set at $1.39 .^{8,28}$ For dysplastic cells, darker appearance of Feulgenthionin-stained nuclei in histology slides indicates higher concentrations of nucleic acids and nucleoproteins, which leads to higher refractive indices. ${ }^{8,12,30}$ We chose an average refractive index of 1.42 for the nucleolus and chromatin condensation in models of dysplastic nuclei.

\section{Results}

We verified our 3-D FDTD code by comparing backscattering spectra of homogeneous spheres with theoretical results provided by Mie theory. Figure 1 shows the scattered intensity integrated over the collection cone of the assumed objective lens. We present the spectra using the same unit as the scattering cross section $\left(\mathrm{m}^{2}\right)$, and refer to the results as backscattering cross section. FDTD spectra of homogeneous spheres with diameters of 5 and $8 \mu \mathrm{m}$ and refractive indices of 1.39 and 1.42 match well to values predicted by Mie theory. Sample nuclear models with $1.67-\mu \mathrm{m}$ nucleoli are shown in Fig. 2. Four different chromatin densities were used to account for a variety of nuclear structure. The volume fraction of the nucleolus plus chromatin condensation was 22.1, 23.4, 35.8, and 47.2\%, respectively [(Figs. 2(a)-2(d))].

To separate influences of multiple parameters on the resulting spectra, we first set the refractive index of the nucleolus and chromatin to be constant. Figure 3 shows FDTD results of normal nuclei (circles) and dysplastic nuclei (triangles) using the same structures of nucleoli and chromatin clumps as shown in Fig. 2. Backscattering spectra from heterogeneous spheres show similar oscillations in intensity as those from homogeneous spheres in all cases, including the one with very low chromatin density [Fig. 3(a)]. For calculating the back- 

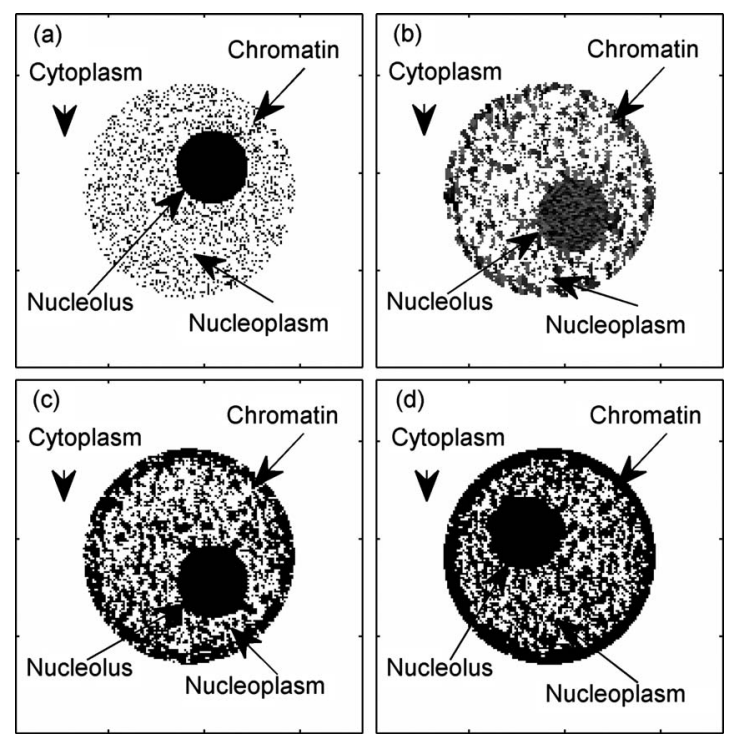

Fig. 2 Illustrations of simulated models of spherical nuclei with increasing chromatin densities from (a) to (d). In (b), refractive index fluctuation is depicted in grayscale. In the other three cases, the nucleolus and chromatin clumps are assumed to have a constant refractive index and are shown in black.

scattering spectra of $5-\mu \mathrm{m}$ homogeneous spheres with Mie theory, we used the average refractive index of the corresponding heterogeneous nucleus as an input parameter. We note several observations from the results. First, although the intensities of the peaks and valleys of heterogeneous spheres differ from those of homogeneous spheres, the wavelengths of the peaks and valleys are almost the same, indicating the feasibility of sizing heterogeneous spheres by comparing their
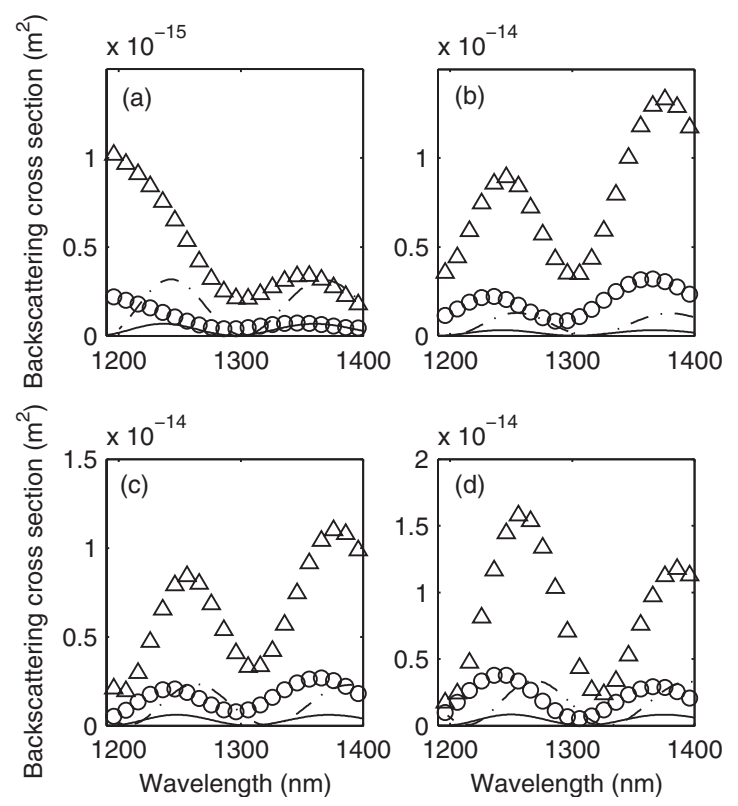

Fig. 3 Backscattering spectra of normal (circles) and dysplastic (triangles) nuclei by FDTD simulations. Spectra of corresponding homogeneous spheres obtained by Mie theory are represented by solid and dotted lines, respectively.
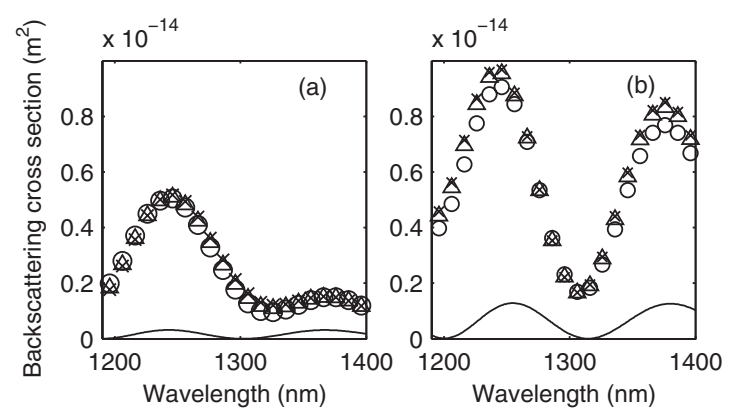

Fig. 4 (a) Backscattering spectra of a normal nucleus calculated by FDTD using the same chromatin condensation distribution and different refractive indices in the ranges of $1.39(\bigcirc), 1.39 \pm 0.004(\triangle)$, and $1.39 \pm 0.007(\times)$. (b) Backscattering spectra of a dysplastic nucleus with refractive indices in the ranges of $1.42(\bigcirc), 1.42 \pm 0.008(\triangle)$, and $1.42 \pm 0.01(\times)$. Backscattering spectra of the corresponding homogeneous spheres by Mie are shown in solid lines.

backscattering spectra with Mie theory. Second, increase in backscattering intensity due to a higher relative refractive index of the dysplastic model is evident for both homogeneous and heterogeneous spheres. Third, heterogeneous spheres show higher scattering intensities than the corresponding homogeneous spheres, presumably because there are more reflecting interfaces in heterogeneous spheres.

Next, we investigated the influence of refractive index fluctuation within the nucleus, which has been shown to increase in dysplastic cells. ${ }^{12}$ The nuclear models were constructed as described before, except that the values of refractive index were randomly assigned within a certain range instead of being a constant. One representative model is shown in Fig. 2(b). Figure 4(a) shows backscattering spectra of a simulated normal nucleus having refractive indices in the ranges of $1.39,1.39 \pm 0.004$, and $1.39 \pm 0.007$. Backscattering spectra of a dysplastic nucleus with refractive indices in the ranges of $1.42,1.42 \pm 0.008$, and $1.42 \pm 0.010$ are shown in Fig. 4(b). Scattering spectra of corresponding homogeneous spheres are shown in solid lines. The results show that refractive index fluctuations in simulated nuclei have no effect on peak and valley wavelengths in backscattering spectra, and only cause slight increases in scattering intensity.

These analyses are based on comparing simulation results between nuclear models having the same structures. However, our results indicate that the spectral characteristics of backscattering are highly dependent on the specific distribution of chromatin condensation in the simulated nucleus. Attempting to capture a general trend in the relation between backscattering spectra and nuclear structure, we simulated 28 normal nuclei and 19 dysplastic nuclei with various parameters. Average FDTD spectra of the normal and dysplastic nuclei are shown in Fig. 5, along with Mie spectra of homogeneous spheres obtained by using the average refractive indices of the corresponding heterogeneous nuclei. The results show trends similar to those seen in Fig. 3. The ratio of maximum intensities between heterogeneous and homogeneous spheres is 6.2 and 4.9 for normal and dysplastic models, respectively.

To assess the accuracy of using Mie theory to estimate the size of heterogeneous spheres, we compared the FDTD result with spectra of homogeneous spheres having various diameters, and determined the best-fit diameter by minimizing the 

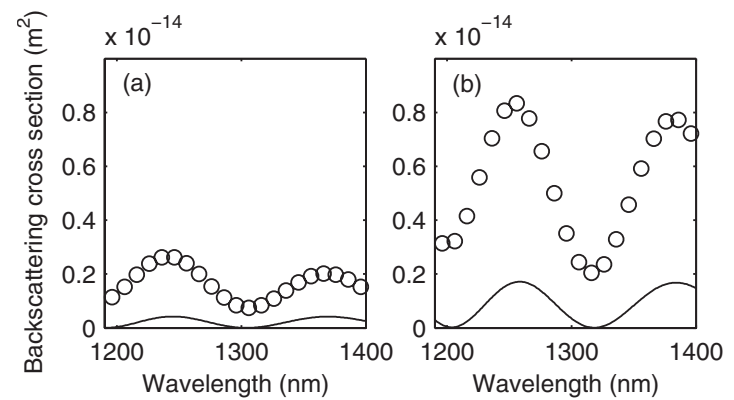

Fig. 5 Average backscattering spectra of simulated (a) normal and (b) dysplastic nuclei $(\bigcirc)$. The average refractive indices of the normal $(n=1.375)$ and dysplastic $(n=1.39)$ nuclei are used to get the spectra of homogeneous spheres (solid lines).

sum of the rms error in intensity, the difference in peak and valley wavelengths, and the difference in peak-to-peak and valley-to-valley distances between normalized backscattering spectra obtained with FDTD and Mie. The result of size estimation is summarized in Fig. 6. The average error in size estimation is $8 \%$, excluding six outliers in which shifts in peak and/or valley wavelengths and the absence of a peak and/or valley results in large errors in the estimated diameters. The results indicate that high accuracy of size estimation is not sensitive to nucleolar diameter, refractive index fluctuation, or chromatin density.

\section{Discussion}

The research reported here focuses on investigating the effects of heterogeneous refractive index distribution of spherical cell nuclei on the spectral characteristics of backscattered light. The results indicate that oscillations in backscattering spectra of modeled heterogeneous nuclei can be used to estimate the size of the nuclei by comparing with Mie theory, which supports in vivo experimental results of previous studies based on light scattering spectroscopy (LSS) ${ }^{3-6}$ In our simulated models of nuclei, clumps of chromatin having higher refractive indices are embedded in a homogeneous medium representing the nucleoplasm and cytoplasm. According to scattering theory, particles the size of cell nuclei contribute more significantly to forward scattering, while backscattering is dominated by particles about the same size as the wavelength or

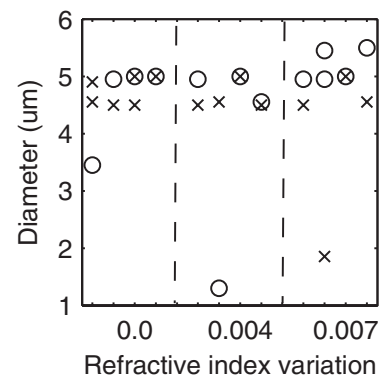

(a)

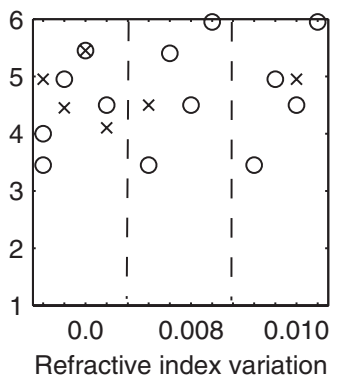

(b)
Fig. 6 Results of estimating the size of (a) normal and (b) dysplastic nuclei. For each refractive index variation range, results with increasing chromatin density are shown from left to right. Nucleolar diameter is either $1.25 \mu \mathrm{m}(\times)$ or $1.67 \mu \mathrm{m}(O)$.
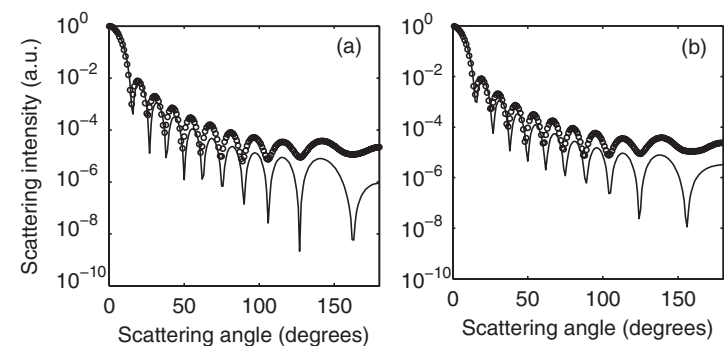

Fig. 7 Angular scattering patterns at a wavelength of $1300 \mathrm{~nm}$ averaged from (a) normal and (b) dysplastic nuclei (circles). Angular scattering patterns of the corresponding Mie spheres are plotted in solid lines for comparison. Scattering intensities are normalized and plotted in log scale.

smaller. ${ }^{1,2,8,11,12}$ Therefore, most of the backscattered light is expected to originate from the subwavelength chromatin clumps. However, our results show that the spectral dependence of backscattering is similar to that of homogeneous spheres several wavelengths in diameter, suggesting that small-sized refractive index variations located closely to each other need to be considered together rather than as independent centers of single-scattering events. A similar correlation between homogeneous and inhomogeneous spheres has been demonstrated in the total scattering cross sectional spectra by using analytical approximation and rigorous broadband FDTD simulation. ${ }^{16,31}$ In these previous studies, the total scattering cross section is dominated by forward scattering, because the spheres are several wavelengths in diameter. The findings of our study on backscattering are significant, because they are directly applicable to the interpretation of reflectance spectral data from in vivo studies.

Another noteworthy result of modeling cell nuclei as heterogeneous spheres is the increase in backscattered intensity by a factor of 5 to 6 over homogeneous counterparts. This result suggests that modeling LSS by simplifying cell nuclei as homogeneous spheres, which is convenient and commonly used in studies for in vivo diagnosis of dysplasia, underestimates the backscattering intensity from nuclei. ${ }^{32}$ While this simplification does not significantly affect the accuracy of sizing nuclei (because experimental measurements of light intensity are usually relative), a more accurate model is necessary if the contribution of backscattering from the nuclei is to be compared with diffuse reflectance for modeling light propagation in tissue. The result of higher backscattering from dysplastic nuclei compared to normal nuclei agrees with previous FDTD studies in which increased backscattering has been attributed to increased mean refractive indices and higher refractive index fluctuations, representing elevated DNA content and higher degrees of heterogeneity in nuclei, respectively. ${ }^{8,12}$

Although the focus of the current study is to investigate the spectral dependence of backscattering from individual cell nuclei, the reported broadband FDTD method also obtains angular distributions of scattered light (Fig. 7). Therefore, it could be incorporated into models of other light spectroscopy techniques for in vivo diagnosis, because the measured signal from tissue is strongly influenced by the scattering properties of tissue. For example, the Monte Carlo method has been widely used to simulate photon propagation in soft tissues. A 
recent study demonstrates using FDTD simulation to generate more realistic phase functions to replace the general HenyeyGreenstein phase function. ${ }^{33}$ The reported broadband FDTD method could extend the approach to wavelength-dependent phase functions for spectroscopic modeling and data analysis using the Monte Carlo method.

For spectroscopic measurement and analysis of elastically backscattered light from bulk tissue, contributions of scattered light from nuclei of different sizes may obscure the oscillating patterns in the measured spectra, hence impeding accurate extraction of the nuclear size distribution. Systems combining high-resolution imaging and spectroscopic capabilities can provide spectral information backscattered from single or a few cells, and are advantageous over spectroscopic measurement of the bulk tissue in estimating the size of nuclei. For instance, systems based on spectroscopic optical coherence microscopy ${ }^{34}$ and spectroscopic confocal microscopy ${ }^{35,36}$ have been demonstrated to obtain backscattering spectra from localized regions within living cells. To what extent and how accurately Mie theory could be applied to estimating the size of diagnostically relevant subcellular structures depends on both the optical arrangement used for data acquisition and the specific structure of the cell being measured. Our current FDTD code uses plane-wave illumination and a moderate NA for the collection objective. Further research attempting to address these questions requires implementing the FDTD method with focused incident beams, ${ }^{37,38}$ and incorporating detection methods such as confocal and coherence gating into the model.

One major limitation of the reported work and other studies using FDTD for modeling biological cells is the lack of detailed 3-D refractive index distribution of living cells. Confocal microscopy can be used to obtain 3-D structures of living cells with proper fluorescence labeling. ${ }^{13}$ However, refractive indices of cellular constituents are not available from confocal imaging. 3-D refractive index mapping of living cells has recently been demonstrated with quantitative phase microscopy in conjunction with tomographic reconstruction. ${ }^{39}$ This technique is very promising for providing the desired refractive index information of living cells. However, due to the limitation in spatial resolution, heterogeneities such as chromatin clumps with a size below the diffraction limit are blurred in reconstructed images and underestimated in refractive index. Since light scattering is sensitive to structures much smaller than the diffraction limit, combining tomographic phase microscopy with light scattering techniques may provide more accurate and detailed 3-D distribution of refractive index in living cells.

It has been suggested that the refractive index variations in biological cells and tissue show self-affine or fractal features based on images of stained cell nuclei in cytological specimens, ${ }^{24}$ and frozen and thawed tissues. ${ }^{23}$ While the fixation processes are likely to alter the structure of specimens, experimental evidences from samples free of staining or fixation, including freshly excised rat tissues ${ }^{25,26}$ and cell monolayers, ${ }^{7,27}$ have been obtained using polarized LSS ${ }^{25,26}$ and angle-resolved low-coherence interferometry (a/LCI). ${ }^{7,27}$ However, fractal dimension in a/LCI is obtained after the angular scattering component of nuclei is removed, assuming that the angular backscattering patterns of heterogeneous nuclei can be represented by those of homogeneous spheres. We note that this assumption is supported by the current study, as shown in Fig. 7. Polarized LSS typically measures bulk tissue with illumination beams several millimeters in diameter, and it is unclear from previously published results how much epithelial cell nuclei contribute to the inverse power law scattering spectra. Further investigations are needed to fully elucidate the origin of the fractal-like features of light scattering from biological tissue and cells.

\section{Conclusions}

We report 3-D implementation of a broadband FDTD method to obtain spectral characteristics of backscattered light from normal and dysplastic cell nuclei, which are modeled as spheres having a spherical nucleolus and randomly distributed chromatin condensation. The resemblance between backscattering spectra from heterogeneous nuclei and those from homogeneous spheres suggests that estimating the size of nuclei based on Mie theory is plausible. Intensity of backscattered light from heterogeneous nuclei is about five times higher than that from homogeneous spheres. Results of this study indicate that small-sized refractive index variations within the nuclei enhance backscattering and collectively exhibit scattering characteristics of the much larger nuclei. Light scattering properties obtained by the broadband FDTD method could be incorporated into Monte Carlo simulations to optimize both the design of optical probes for measuring spectroscopic data from tissue, and the development of algorithms for extracting optical properties of the tissue from measured data.

\section{Acknowledgments}

The authors thank the National Science Council for financial support (grants 95-2218-E-002-063 and 98-2112-M-002-009) of this research, and Dr. Dizem Arifler for providing advice on the implementation of the FDTD code.

\section{References}

1. J. R. Mourant, J. P. Freyer, A. H. Hielscher, A. A. Eick, D. Shen, and T. M. Johnson, "Mechanisms of light scattering from biological cells relevant to noninvasive optical-tissue diagnostics," Appl. Opt. 37(16), 3586-3593 (1998).

2. J. R. Mourant, T. M. Johnson, S. Carpenter, A. Guerra, T. Aida, and J. P. Freyer, "Polarized angular dependent spectroscopy of epithelial cells and epithelial cell nuclei to determine the size scale of scattering structures," J. Biomed. Opt. 7(3), 378-387 (2002).

3. L. T. Perelman, V. Backman, M. Wallace, G. Zonios, R. Manoharan, A. Nusrat, S. Shields, M. Seiler, C. Lima, T. Hamano, I. Itzkan, J. Van Dam, J. M. Crawford, and M. S. Feld, "Observation of periodic fine structure in reflectance from biological tissue: a new technique for measuring nuclear size distribution," Phys. Rev. Lett. 80(3), 627630 (1998).

4. V. Backman, R. Gurjar, K. Badizadegan, L. Itzkan, R. R. Dasari, L. T. Perelman, and M. S. Feld, "Polarized light scattering spectroscopy for quantitative measurement of epithelial cellular structures in situ," IEEE J. Sel. Top. Quantum Electron. 5(4), 1019-1026 (1999).

5. V. Backman, M. B. Wallace, L. T. Perelman, J. T. Arendt, R. Gurjar, M. G. Muller, Q. Zhang, G. Zonios, E. Kline, T. McGillican, S. Shapshay, T. Valdez, K. Badizadegan, J. M. Crawford, M. Fitzmaurice, S. Kabani, H. S. Levin, M. Seiler, R. R. Dasari, I. Itzkan, J. Van Dam, and M. S. Feld, "Detection of preinvasive cancer cells," Nature 406(6791), 35-36 (2000).

6. K. Sokolov, R. Drezek, K. Gossage, and R. Richards-Kortum, "Reflectance spectroscopy with polarized light: is it sensitive to cellular and nuclear morphology," Opt. Express 5(13), 302-317 (1999).

7. A. Wax, C. H. Yang, V. Backman, K. Badizadegan, C. W. Boone, R. 
R. Dasari, and M. S. Feld, "Cellular organization and substructure measured using angle-resolved low-coherence interferometry," Biophys. J. 82(4), 2256-2264 (2002).

8. R. Drezek, M. Guillaud, T. Collier, I. Boiko, A. Malpica, C. Macaulay, M. Follen, and R. Richards-Kortum, "Light scattering from cervical cells throughout neoplastic progression: influence of nuclear morphology, DNA content, and chromatin texture," $J$. Biomed. Opt. 8(1), 7-16 (2003).

9. C. Mujat, C. Greiner, A. Baldwin, J. M. Levitt, F. Tian, L. A. Stucenski, M. Hunter, Y. L. Kim, V. Backman, M. Feld, K. Muenger, and I. Georgakoudi, "Endogenous optical biomarkers of normal and human papillomavirus immortalized epithelial cells," Int. J. Cancer 122(2), 363-371 (2008).

10. A. Dunn and R. Richards-Kortum, "Three-dimensional computation of light scattering from cells," IEEE J. Sel. Top. Quantum Electron. 2(4), 898-905 (1996).

11. R. Drezek, A. Dunn, and R. Richards-Kortum, "Light scattering from cells: finite-difference time-domain simulations and goniometric measurements," Appl. Opt. 38(16), 3651-3661 (1999).

12. D. Arifler, M. Guillaud, A. Carraro, A. Malpica, M. Follen, and R. Richards-Kortum, "Light scattering from normal and dysplastic cervical cells at different epithelial depths: finite-difference time-domain modeling with a perfectly matched layer boundary condition," $J$. Biomed. Opt. 8(3), 484-494 (2003).

13. R. S. Brock, X. H. Hu, D. A. Weidner, J. R. Mourant, and J. Q. Lu, "Effect of detailed cell structure on light scattering distribution: FDTD study of a B-cell with 3D structure constructed from confocal images," J. Ouant. Spectrosc. Radiat. Transf. 102(1), 25-36 (2006).

14. X. T. Su, C. Capjack, W. Rozmus, and C. Backhouse, "2D light scattering patterns of mitochondria in single cells," Opt. Express 15(17), 10562-10575 (2007).

15. R. Drezek, A. Dunn, and R. Richards-Kortum, "A pulsed finitedifference time-domain (FDTD) method for calculating light scattering from biological cells over broad wavelength ranges," Opt. Express $6(7), 147-157$ (2000).

16. X. Li, Z. G. Chen, A. Taflove, and V. Backman, "Equiphase-sphere approximation for light scattering by stochastically inhomogeneous microparticles," Phys. Rev. E 70(5), 056610 (2004).

17. X. Li, Z. G. Chen, J. M. Gong, A. Taflove, and V. Backman, "Analytical techniques for addressing forward and inverse problems of light scattering by irregularly shaped particles," Opt. Lett. 29(11), 1239-1241 (2004).

18. X. Li, A. Taflove, and V. Backman, "Recent progress in exact and reduced-order modeling of light-scattering properties of complex structures," IEEE J. Sel. Top. Quantum Electron. 11(4), 759-765 (2005).

19. K. B. Sung, R. Richards-Kortum, M. Follen, A. Malpica, C. Liang, and M. R. Descour, "Fiber optic confocal reflectance microscopy: a new real-time technique to view nuclear morphology in cervical squamous epithelium in vivo," Opt. Express 11(24), 3171-3181 (2003).

20. K. S. Yee, "Numerical solution of initial boundary value problems involving Maxwell's equations in isotropic media," IEEE Trans. Antennas Propag. AP14(3), 302-307 (1966).

21. J. P. Berenger, "Three-dimensional perfectly matched layer for the absorption of electromagnetic waves," J. Comput. Phys. 127(2), 363 379 (1996)

22. D. W. Fawcett, "Nucleus," Chap. 4 in The Cell, pp. 195-292, W. B. Saunders Co., West Washington Square Philadelphia, PA (1981).

23. J. M. Schmitt and G. Kumar, "Turbulent nature of refractive-index variations in biological tissue," Opt. Lett. 21(16), 1310-1312 (1996).

24. A. J. Einstein, H. S. Wu, and J. Gil, "Self-affinity and lacunarity of chromatin texture in benign and malignant breast epithelial cell nuclei," Phys. Rev. Lett. 80(2), 397-400 (1998).

25. Y. L. Kim, Y. Liu, R. K. Wali, H. K. Roy, M. J. Goldberg, A. K. Kromin, K. Chen, and V. Backman, "Simultaneous measurement of angular and spectral properties of light scattering for characterization of tissue microarchitecture and its alteration in early precancer," IEEE J. Sel. Top. Quantum Electron. 9(2), 243-256 (2003).

26. M. Hunter, V. Backman, G. Popescu, M. Kalashnikov, C. W. Boone, A. Wax, V. Gopal, K. Badizadegan, G. D. Stoner, and M. S. Feld, "Tissue self-affinity and polarized light scattering in the Born approximation: A new model for precancer detection," Phys. Rev. Lett. 97(13), 138102 (2006).

27. K. J. Chalut, J. H. Ostrander, M. G. Giacomelli, and A. Wax, "Light scattering measurements of subcellular structure provide noninvasive early detection of chemotherapy-induced apoptosis," Cancer Res. 69(3), 1199-1204 (2009).

28. A. Brunstin and P. F. Mullaney, "Differential light-scattering from spherical mammalian-cells," Biophys. J. 14(6), 439-453 (1974).

29. K. E. Handwerger, J. A. Cordero, and J. G. Gall, "Cajal bodies, nucleoli, and speckles in the Xenopus oocyte nucleus have a lowdensity, sponge-like structure," Mol. Biol. Cell 16(1), 202-211 (2005).

30. R. Barer, "Refractometry and interferometry of living cells," J. Opt. Soc. Am. A 47(6), 545-556 (1957).

31. Z. G. Chen, A. Taflove, and V. Backman, "Equivalent volumeaveraged light scattering behavior of randomly inhomogeneous dielectric spheres in the resonant range," Opt. Lett. 28(10), 765-767 (2003).

32. C. Lau, O. Scepanovic, J. Mirkovic, S. McGee, C. C. Yu, S. Fulghum, M. Wallace, J. Tunnell, K. Bechtel, and M. Feld, "Reevaluation of model-based light-scattering spectroscopy for tissue spectroscopy," J. Biomed. Opt. 14(2), 024031 (2009).

33. C. Kortun, Y. R. Hijazi, and D. Arifler, "Combined Monte Carlo and finite-difference time-domain modeling for biophotonic analysis: implications on reflectance-based diagnosis of epithelial precancer," $J$. Biomed. Opt. 13(3), 034014 (2008).

34. C. Y. Xu, C. Vinegoni, T. S. Ralston, W. Luo, W. Tan, and S. A. Boppart, "Spectroscopic spectral-domain optical coherence microscopy," Opt. Lett. 31(8), 1079-1081 (2006).

35. I. Itzkan, L. Qiu, H. Fang, M. M. Zaman, E. Vitkin, L. C. Ghiran, S. Salahuddin, M. Modell, C. Andersson, L. M. Kimerer, P. B. Cipolloni, K. H. Lim, S. D. Freedman, I. Bigio, B. P. Sachs, E. B. Hanlon, and L. T. Perelman, "Confocal light absorption and scattering spectroscopic microscopy monitors organelles in live cells with no exogenous labels," Proc. Natl. Acad. Sci. U.S.A. 104(44), 17255-17260 (2007).

36. P. Huang, M. Hunter, and I. Georgakoudi, "Confocal light scattering spectroscopic imaging system for in situ tissue characterization," Appl. Opt. 48(13), 2595-2599 (2009).

37. I. R. Capoglu, A. Taflove, and V. Backman, "Generation of an incident focused light pulse in FDTD," Opt. Express 16(23), 1920819220 (2008).

38. M. S. Starosta and A. K. Dunn, "Three-dimensional computation of focused beam propagation through multiple biological/Cells," Opt. Express 17(15), 12455-12469 (2009).

39. Y. J. Sung, W. Choi, C. Fang-Yen, K. Badizadegan, R. R. Dasari, and M. S. Feld, "Optical diffraction tomography for high resolution live cell imaging," Opt. Express 17(1), 266-277 (2009). 